\title{
Systematic study of real photon and Drell-Yan pair production in $p+A(d+A)$ interactions
}

\author{
M. Krelina ${ }^{1, a}$, E. Basso ${ }^{2,3}$, V. P. Goncalves ${ }^{3,4}$, J. Nemchik ${ }^{1,5}$, and R. Pasechnik ${ }^{3}$ \\ ${ }^{1}$ FNSPE, Czech Technical University in Prague, Brehova 7, 11519 Prague, Czech Republic \\ ${ }^{2}$ Instituto de FIsica, Universidade Federal do Rio de Janeiro, Caixa Postal 68528, Rio de Janeiro, RJ 21941- \\ 972, Brazil \\ ${ }^{3}$ Department of Astronomy and Theoretical Physics, Lund University, SE-223 62 Lund, Sweden \\ ${ }^{4}$ High and Medium Energy Group, Instituto de Fisica e Matematica, Universidade Federal de Pelotas, \\ Pelotas, RS, 96010-900, Brazil \\ ${ }^{5}$ Institute of Experimental Physics SAS, Watsonova 47, 04001 Kosice, Slovakia
}

\begin{abstract}
We study nuclear effects in production of Drell-Yan pairs and direct photons in proton-nucleus collisions. For the first time, these effects are studied within the color dipole approach using the Green function formalism which naturally incorporates the color transparency and quantum coherence effects. The corresponding numerical results for the nuclear modification factor are compared with available data. Besides, we present a variety of predictions for the nuclear suppression as a function of transverse momentum $p_{T}$, Feynman variable $x_{F}$ and invariant mass $M$ of the lepton pair which can be verified by experiments at RHIC and LHC. We found that the nuclear suppression is caused predominantly by effects of quantum coherence (shadowing corrections) and by the effective energy loss induced by multiple initial state interactions. Whereas the former dominate at small Bjorken $x_{2}$ in the target, the latter turns out to be significant at large $x_{1}$ in the projectile beam and is universal at different energies and transverse momenta.
\end{abstract}

\section{Introduction}

The color dipole approach [1] represents a phenomenological framework that effectively takes into account the higher-order and nonlinear QCD effects. There are many studies in the literature demonstrating a reliable agreement of predictions with experimental data, especially at high energies and/or small Bjorken variable $x_{2}$ in proton-proton $(p p)$ collisions and DIS (see e.g. Refs. [2-4] and references therein).

The color dipole approach which is formulated in the target rest frame provides a consistent way of studying the nuclear effects, especially the nuclear shadowing, in both proton-nucleus $(p A)$ and nucleus-nucleus $(A A)$ collisions. The dynamics of $p A$ or $A A$ collisions is controlled by the coherence length $l_{c}$. When the coherence length is sufficiently large or small, one talks about the long coherence length (LCL) or short coherence length (SCL) approximations, respectively. In the intermediate kinematics when both approximations fail, one should employ the Green function technique which

\footnotetext{
a e-mail: michal.krelina@fjfi.cvut.cz
} 
accounts for the exact coherence length $l_{c}$ and naturally incorporates the color transparency and quantum coherence effects. Such a kinematic region corresponds e.g. to kinematics at RHIC fixed target experiments or planned experiments such as AFTER@LHC.

In this paper, we present numerical results on the quark-nucleus cross section within the Green function formalism for the Drell-Yan (DY) lepton pair production and production of direct photons. Besides, we include also the gluon shadowing (GS) that dominates at small Bjorken $x_{2}$ and the effective energy loss induced by multiple initial state interactions.

\section{Coherence length}

The rest frame of the nucleus is very convenient for study of coherence effects. The dynamics of DrellYan (DY) process is regulated by the coherence length $l_{c}$, which controls the interference between amplitudes of the hard reaction occurring on different nucleons and is given by

$$
l_{c}=\frac{1}{x_{2} m_{N}} \frac{\left(M^{2}+p_{T}^{2}\right)(1-\alpha)}{\alpha(1-\alpha) M^{2}+\alpha^{2} m_{f}^{2}+p_{T}^{2}},
$$

where $\alpha$ is the fraction of the light-cone momentum of the projectile quark carried out by the photon, and $m_{q}=0.2 \mathrm{GeV}$ is an effective quark mass. Figs. 1 and 2 show the energy dependence of the mean coherence length for $x_{F}=0$ and $x_{F}=0.6$ corresponding to small $x_{2}$ fractions, explicitly separating the regimes with the long coherence length (LCL), $l_{c}>R_{A}$, and short coherence length (SCL), $l_{c} \lesssim 1 \div 2 \mathrm{fm}$. For the transition region between both limits we used the Green function formalism as the general case with no restrictions on $l_{c}$.

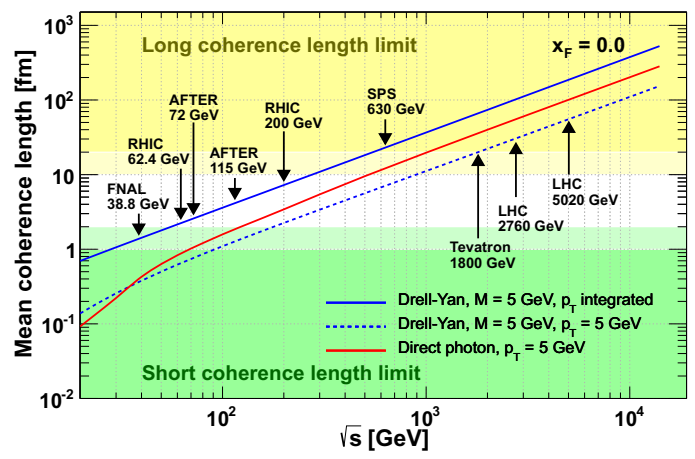

Figure 1. The mean coherence length for Drell-Yan and direct photons production for $x_{F}=0$.

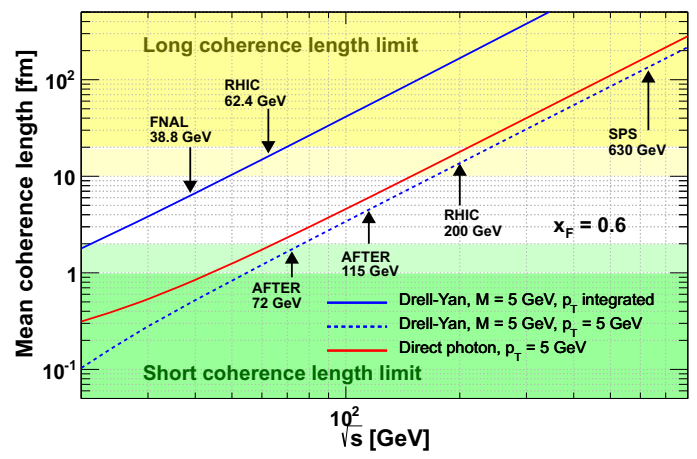

Figure 2. The mean coherence length for Drell-Yan and direct photons production for $x_{F}=0.6$.

\section{Color dipole approach}

The DY process in the target rest frame can be treated as a radiation of a heavy photon or $Z^{0}$ boson by a projectile quark. The transverse momentum $p_{T}$ distribution of photon bremsstrahlung in quarknucleon interactions reads [5]

$$
\frac{d^{3} \sigma^{\left(q N \rightarrow \gamma^{*} X\right)}}{d \ln \alpha d^{2} p_{T}}=\frac{1}{(2 \pi)^{2}} \int d^{2} \rho_{1} d^{2} \rho_{2} e^{i \vec{p}_{T} \cdot\left(\vec{\rho}_{1}-\vec{\rho}_{2}\right)} \Psi_{\gamma^{*} q}^{*}\left(\alpha, \vec{\rho}_{2}\right) \Psi_{\gamma^{*} q}\left(\alpha, \vec{\rho}_{1}\right) \Sigma\left(\alpha, \vec{\rho}_{1}, \vec{\rho}_{2}\right),
$$


where

$$
\Sigma\left(\alpha, \vec{\rho}_{1}, \vec{\rho}_{2}\right)=\frac{1}{2}\left(\sigma_{q \bar{q}}^{N}\left(\alpha \vec{\rho}_{1}\right)+\sigma_{q \bar{q}}^{N}\left(\alpha \vec{\rho}_{2}\right)-\sigma_{q \bar{q}}^{N}\left(\alpha\left(\vec{\rho}_{1}-\vec{\rho}_{2}\right)\right)\right)
$$

and the light-cone (LC) wave functions of the projectile $q \rightarrow q+\gamma$ fluctuation $\Psi_{T, L}(\alpha, \vec{\rho})$ can be found in Ref. [5]. For the dipole cross section $\sigma_{q \bar{q}}^{N}(\alpha \vec{\rho})$ we used GBW [6], KST [7] and GBWnew [8] parameterisations. The hadron cross section is given by a convolution of the $q N$ cross section with the corresponding parton distribution functions (PDFs) $f_{q}$ and $\bar{f}_{q}$ as follows

$$
\frac{d^{4} \sigma^{\left(p p \rightarrow l^{+} l^{-} X\right)}}{d^{2} p_{T} d x_{F} d M^{2}}=\frac{\alpha_{\mathrm{EM}}}{3 \pi^{2}} \frac{x_{1}}{x_{1}+x_{2}} \int_{x_{1}}^{1} \frac{d \alpha}{\alpha^{2}} \sum_{q} Z_{q}\left(q_{f}\left(x_{1} / \alpha, Q^{2}\right)+\bar{q}_{f}\left(x_{1} / \alpha, Q^{2}\right)\right) \frac{d^{3} \sigma^{\left(q N \rightarrow \gamma^{*} X\right)}}{d \ln \alpha d^{2} p_{T}}
$$

where $Z_{q}$ is the fractional quark charge, the (anti)quark PDFs $f_{q}$ and $\bar{f}_{q}$ are used with the leading order (LO) parameterisation from Ref. [9] at the scale $Q^{2}=p_{T}^{2}+\left(1-x_{1}\right) M^{2}$. After integration over the transverse momentum $\vec{p}_{T}$ we get for hadronic cross section

$$
\frac{d^{2} \sigma^{\left(p p \rightarrow l^{+} l^{-} X\right)}}{d x_{F} d M^{2}}=\frac{\alpha_{\mathrm{EM}}}{3 \pi^{2}} \frac{x_{1}}{x_{1}+x_{2}} \int_{x_{1}}^{1} \frac{d \alpha}{\alpha^{2}} \sum_{q} Z_{q}\left(q_{f}\left(x_{1} / \alpha, Q^{2}\right)+\bar{q}_{f}\left(x_{1} / \alpha, Q^{2}\right)\right) \frac{d \sigma^{\left(q N \rightarrow \gamma^{*} X\right)}}{d \ln \alpha}
$$

and for quark-nucleon cross section

$$
\frac{d \sigma^{\left(q N \rightarrow \gamma^{*} X\right)}}{d \ln \alpha}=\int d^{2} \rho\left|\Psi_{\gamma^{*} q}(\alpha, \vec{\rho})\right|^{2} \sigma_{q \bar{q}}^{N}(\alpha \vec{\rho}) .
$$

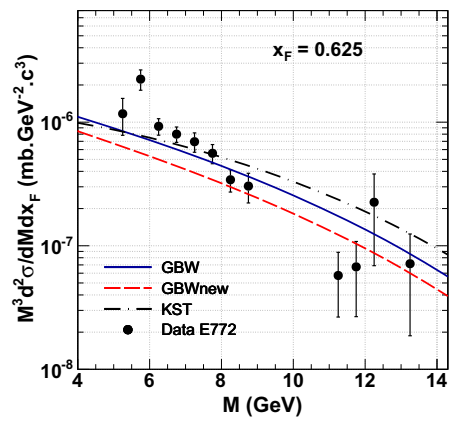

Figure 3. Differential dilepton cross sections in $p p$ collisions vs E772 [13].

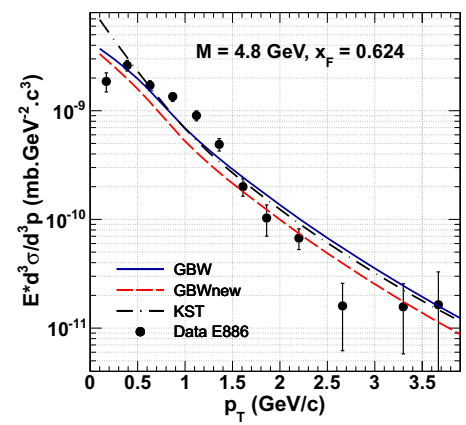

Figure 4. Differential dilepton cross sections in $p p$ collisions vs E886 [14].

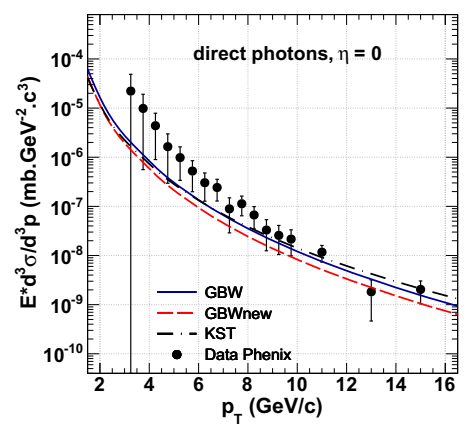

Figure 5. Differential direct photon cross sections in $p p$ collisions vs PHENIX [15].

In Figs. 3, 4 and 5 we compare our predictions for various dipole cross section parameterisations with available DY data from E772 and E886 experiments and for direct photons from the PHENIX experiment where the dipole model predictions agree with the data reasonably well. 


\section{Transition to nuclear target}

Within the Green function formalism the quark-nucleus cross section for DY pair production on nuclear targets reads [5]

$$
\begin{aligned}
\frac{d \sigma^{\left(q A \rightarrow \gamma^{*} X\right)}}{d \ln \alpha} & =A \frac{d \sigma^{\left(q N \rightarrow \gamma^{*} X\right)}}{d \ln \alpha}-\frac{1}{2} \operatorname{Re} \int_{-\infty}^{\infty} d z_{1} \int_{-\infty}^{z_{1}} d z_{2} \int d^{2} b d^{2} \rho_{1} d^{2} \rho_{2} \\
& \times \Psi_{\gamma^{*} q}^{*}\left(\alpha, \vec{\rho}_{2}\right) \rho_{A}\left(b, z_{2}\right) \sigma_{q \bar{q}}^{N}\left(\alpha \vec{\rho}_{2}\right) G\left(\vec{\rho}_{2}, z_{2} \mid \vec{\rho}_{1}, z_{1}\right) \rho_{A}\left(b, z_{1}\right) \sigma_{q \bar{q}}^{N}\left(\alpha \vec{\rho}_{1}\right) \Psi_{\gamma^{*} q}\left(\alpha, \vec{\rho}_{1}\right),
\end{aligned}
$$

where the Green function $G\left(\vec{\rho}_{2}, z_{2} \mid \vec{\rho}_{1}, z_{1}\right)$ describes the propagation of $\left|\gamma^{*} q\right\rangle$ Fock state between longitudinal positions $z_{1}$ and $z_{2}$ through the nucleus with initial and final separations $\vec{\rho}_{1}$ and $\vec{\rho}_{2}$, respectively. The Green function above satisfies the two-dimensional time-dependent Schroedinger equation $\left(z_{2}\right.$ plays the role of time)

$$
\left[i \frac{\partial}{\partial z_{2}}+\frac{\Delta_{T}\left(\vec{\rho}_{2}\right)-\eta^{2}}{2 E_{q} \alpha(1-\alpha)}-V\left(z_{2}, \vec{\rho}_{2}, \alpha\right)\right] G\left(\vec{\rho}_{2}, z_{2} \mid \vec{\rho}_{1}, z_{1}\right)=0
$$

with the boundary condition $\left.G\left(\vec{\rho}_{2}, z_{2} \mid \vec{\rho}_{1}, z_{1}\right)\right|_{z_{1}=z_{2}}=\delta^{2}\left(\vec{\rho}_{2}-\vec{\rho}_{1}\right)$. The imaginary part of the potential $V\left(z_{2}, \vec{\rho}_{2}, \alpha\right)$ describes an absorption of the dipole in a nuclear medium and reads

$$
V\left(z_{2}, \vec{\rho}, \alpha\right)=-\frac{i}{2} \rho_{A}\left(b, z_{2}\right) \sigma_{q \bar{q}}^{N}(\alpha \vec{\rho}) .
$$

For the $p_{T}$-dependent DY production cross section we solved the Schroedinger equation analytically which is possible for quadratic $\sigma_{q \bar{q}}^{N}(\rho)=C \rho^{2}$ and the uniform nuclear density. For $p_{T}$-integrated DY production cross section we solved the Schroedinger equation numerically using an algorithm proposed in Ref. [10].

In the LCL limit the Green function formalism naturally leads to a simple modification of the dipole cross section:

$$
\sigma_{q \bar{q}}^{N}(\vec{\rho}, x) \rightarrow \sigma_{q \bar{q}}^{A}(\vec{\rho}, x)=2 \int d^{2} b\left(1-e^{-\frac{1}{2} \sigma_{q \bar{q}}^{N}(\vec{\rho}, x) T_{A}(b)}\right) .
$$

Besides the lowest $\left|q G^{*}\right\rangle$ Fock state one should include also the higher Fock components containing gluons $\left|\gamma^{*} q G\right\rangle,\left|\gamma^{*} q 2 G\right\rangle$ etc. They cause an additional suppression known as the gluon shadowing (GS). The corresponding suppression factor $R_{G}$ [11] calculated as a correction to the total $\gamma^{*} A$ cross section for the longitudinal photon, $R_{G}\left(x, Q^{2}, b\right) \approx 1-\frac{\Delta \sigma_{L}^{\left(\gamma^{*} A\right)}}{\sigma_{t o t}^{\left(\gamma^{*}\right)}}$, was included in calculations replacing $\sigma_{q \bar{q}}^{N}(\vec{\rho}, x) \rightarrow \sigma_{q \bar{q}}^{N}(\vec{\rho}, x) R_{G}\left(x, Q^{2}, b\right)$.

The initial state energy loss (due to ISI effects) is expected to suppress the nuclear cross section significantly towards the kinematical limits, $x_{L}=\frac{2 p_{L}}{\sqrt{s}} \rightarrow 1$ and $x_{T}=\frac{2 p_{T}}{\sqrt{s}} \rightarrow 1$. Correspondingly, the proper variable which controls this effect is $\xi=\sqrt{x_{L}^{2}+x_{T}^{2}}$. The magnitude of suppression was evaluated in Ref. [12]. It was found within the Glauber approximation that each interaction in the nucleus leads to a suppression factor $S(\xi) \approx 1-\xi$. Summing up over the multiple initial state interactions in a $p A$ collision at impact parameter $b$, one arrives at the nuclear ISI-modified quark PDF

$$
q_{f}\left(x, Q^{2}\right) \Rightarrow q_{f}^{A}\left(x, Q^{2}, b\right)=C_{v} q_{f}\left(x, Q^{2}\right) \frac{e^{-\xi \sigma_{e f f} T_{A}(b)}-e^{-\sigma_{e f f} T_{A}(b)}}{(1-\xi)\left(1-e^{-\sigma_{e f f} T_{A}(b)}\right)} .
$$

Here, $\sigma_{e f f}=20 \mathrm{mb}$ is the effective hadronic cross section controlling the multiple interactions. The normalisation factor $C_{v}$ is fixed by the Gottfried sum rule (for more details, see Ref. [12]), $T_{A}(b)$ 
is the nuclear thickness function at given impact parameter $b$ normalized to the mass number $A$. It was found that such an additional nuclear suppression due to the ISI effects represents an energy independent feature common for all known reactions, experimentally studied so far, with any leading particle (hadrons, Drell-Yan dileptons, charmonium etc).

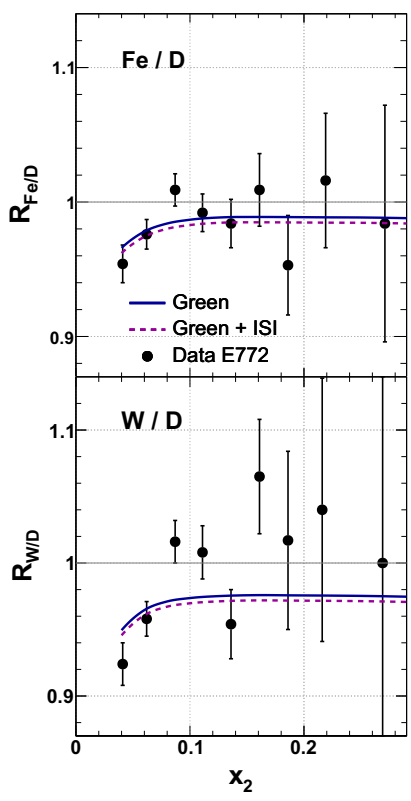

Figure 6. Comparison of the dipole model predictions for $R_{A / B}\left(x_{2}\right)$ with the E772 data at $\sqrt{s}=$ $38.8 \mathrm{GeV}[13]$.

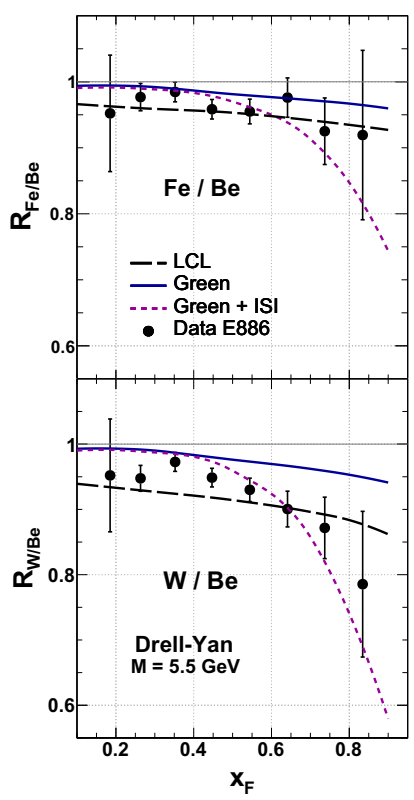

Figure 7. Comparison of the dipole model predictions for $R_{A / B}\left(x_{F}\right)$ with the E886 data at $\sqrt{s}=$ $38.8 \mathrm{GeV}[14]$.

In Figs. 6 and 7 we compare our predictions for ratios $R_{A / B}\left(x_{2}\right)$ and $R_{A / B}\left(x_{F}\right)$ with the E772 and E886 data where the GS is not expected. We obtain a reasonable agreement with the E886 data including the ISI effects. In Fig. 8 we present our predictions for the nuclear suppression of DY pairs production at the future AFTER@LHC experiment demonstrating separate contributions from the GS and ISI effects. Fig. 9 shows the difference between calculations using the Green function formalism and the LCL limit in the RHIC kinematics region for production of direct photons and DY pairs at midrapidity. The RHIC data [15] indicate a strong large- $p_{T}$ suppression that can be explained only by the ISI effects.

\section{Conclusions}

For the first time, we use the Green function formalism based on the color dipole approach for description of DY pair and direct photon production on nuclear targets in the kinematic regions where the SCL and LCL limits should not be used. We demonstrate that the GS and ISI energy loss causes a significant nuclear suppression. While the GS dominates at large energies and $p_{T}$, the ISI effects are important at large $p_{T}$ and/or $x_{F}$. Our predictions are in a good agreement with FNAL E772 and E886 data as well as with the data from the PHENIX Collaboration. Finally, we predict a strong suppression due to the ISI effects that can be verified by the AFTER@LHC experiment in the future. 


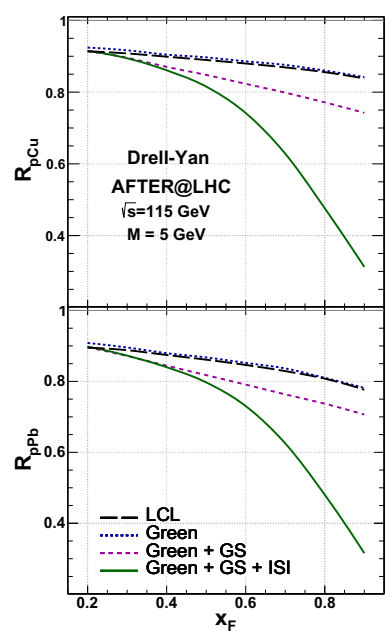

Figure 8. Predictions for the nuclear suppression $R_{p A}$ in the DY process for the AFTER@LHC experiment.

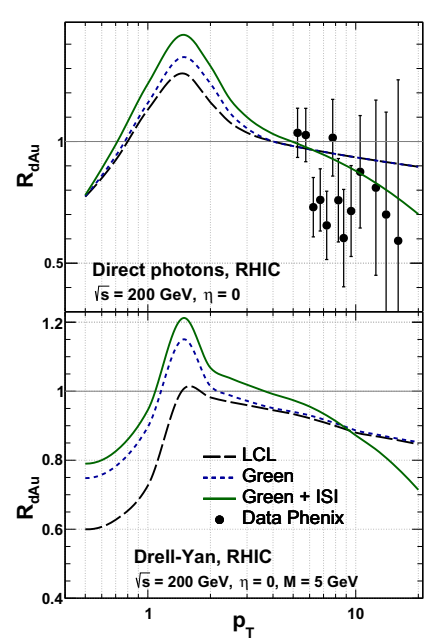

Figure 9. Comparison of $R_{p A}$ with the data from RHIC [15] for direct photons and for the DY process with $M=5 \mathrm{GeV}$.

\section{Acknowledgements}

E. B. is supported by CAPES and CNPq (Brazil), contract numbers 2362/13-9 and 150674/20155. V. P. G. has been supported by CNPq, CAPES and FAPERGS, Brazil. R. P. is supported by the Swedish Research Council, contract number 621-2013-428. J. N. is partially supported by the grant 13-20841S of the Czech Science Foundation (GAČR), by the Grant MŠMT LG13031, by the Grant MŠMT LG15001, by the Slovak Research and Development Agency APVV-0050-11 and by the Slovak Funding Agency, Grant 2/0020/14.

\section{References}

[1] N. N. Nikolaev and B. G. Zakharov, Z. Phys. C64, 631 (1994).

[2] E. Basso, V. P. Goncalves, J. Nemchik, R. Pasechnik, M. Sumbera, arXiv:1510.00650 [hep-ph].

[3] B. Z. Kopeliovich, E. Levin, A. H. Rezaeian and I. Schmidt, Phys. Lett. B675, 190-195 (2009).

[4] B. Z. Kopeliovich, A. H. Rezaeian, H. J. Pirner and I. Schmidt, Phys. Lett. B653, 210-215 (2007).

[5] B. Z. Kopeliovich, A. V. Tarasov and A. Schafer, Phys. Rev. C59, 1609-1619 (1999).

[6] K. Golec-Biernat and M. Wüsthoff, Phys. Rev. D59, 014017 (1998).

[7] B. Z. Kopeliovich, A. Schafer and A. V.Tarasov, Phys. Rev. D62, 054022 (2000).

[8] H. Kowalski, L. Motyka and G. Watt, Phys. Rev. D74, 074016 (2006).

[9] A. D. Martin, W. J. Stirling, R. S. Thorne, G. Watt, Eur. Phys. J. C63, 189 (2009).

[10] J. Nemchik, Phys. Rev. C68, 035206 (2003).

[11] B. Z. Kopeliovich, J. Nemchik and A. Schafer, Phys. Rev. C65, 035201 (2002).

[12] B. Z. Kopeliovich et al., Int. J. Mod. Phys. E23, 1430006 (2014).

[13] D. M. Alde et al. [E772 Collaboration], Phys. Rev. Lett. 64, 2479 (1990).

[14] M. A. Vasiliev et al. [E886 Collaboration], Phys. Rev. Lett. 83, 2304 (1999).

[15] S. S. Adler et al. [PHENIX Collaboration], Phys. Rev. Lett. 98, 012002 (2007). 